\title{
Prepublication
}

Feero, M. A., Lavoie, P., \& Sullivan, P. E. (2014). Influence of Cavity Shape on Synthetic Jet Performance. Sensors and Actuators A: Physical.

doi:10.1016/j.sna.2014.12.004

\section{Influence of Cavity Shape on Synthetic Jet}

\section{Performance}

\author{
Mark A. Feero*, Philippe Lavoie ${ }^{\dagger}$ and Pierre E. Sullivan ${ }^{\ddagger}$ \\ University of Toronto, Toronto, Ontario, Canada
}

\begin{abstract}
A synthetic jet is an unsteady jet that transfers linear momentum to the surroundings by alternately ingesting and expelling fluid from a cavity containing an oscillating diaphragm. This work presents the first experimental effort to validate the limited number of numerical investigations that have postulated synthetic jets are insensitive to cavity shape. Three axisymmetric synthetic jets with different cavity shapes were used to examine jet performance while keeping other parameters constant such as cavity volume, nozzle length and orifice diameter. Cylindrical, conical and contraction shaped cavities were considered. The cavity pressure and velocity at the orifice exit plane were measured using a microphone and hot-wire, respectively. The results demonstrated that for several operating conditions near Helmholtz resonance of the cavity, noticeable differences were observed in the radial velocity profiles between the three geometries. The Reynolds number decreased sequentially from the cylindrical to conical to contraction cavity. The momentum flux, which is relevant in flow control applications, followed the same trend. In general, the experimental results showed that synthetic jet performance is, to some degree, dependant on cavity shape.
\end{abstract}

Keywords: Synthetic jet, Fluidic actuator, Cavity shape

*PhD Student (corresponding author), Institute for Aerospace Studies, 4925 Dufferin Street, Toronto, Ontario, M3H 5T6,

Canada, m.feero@mail.utoronto.ca, +1 (416)667-7716

${ }^{\dagger}$ Professor, Institute for Aerospace Studies, lavoie@utias.utoronto.ca

${ }^{\ddagger}$ Professor, Mechanical and Industrial Engineering, sullivan@mie.utoronto.ca 


\section{Introduction}

Synthetic jet actuators have proven to be useful for a number of applications including cooling of electronic devices $[18,12]$ and flow control on aerodynamic bodies $[1,9]$. Compared with continuous or pulsed jets, synthetic jets offer a distinct advantage in that they require no external fluid source. A synthetic jet actuator is composed of an orifice or slot connected to a cavity in which one or more surfaces oscillates. As the wall(s) of the cavity oscillate(s), the working fluid is alternately ingested and expelled through the orifice, forming a train of discrete vortical structures that transfer linear momentum to the flow without net mass injection [8]. The fact that no external fluid source is required combined with the availability of increasingly small vibrating diaphragms (e.g. piezoelectric disks) allows the design of extremely compact devices, even down to MEMS scales (e.g. [14]). Despite the widespread usage of synthetic jets, the complex dynamic behaviour of these devices remains a topic of research.

The characteristic velocity of a synthetic jet is often taken as the centreline velocity, $U_{c l}$, due to the assumption of a simple top-hat velocity profile at the orifice/slot exit. Since the spatial velocity profile can show significant deviation from the top-hat shape, a more general velocity scale is the time-and-spatiallyaveraged exit velocity over the expulsion stroke, viz.

$$
\bar{U}=\frac{1}{T / 2} \frac{1}{A_{n}} \int_{A_{n}} \int_{0}^{T / 2} U\left(t, A_{n}\right) \mathrm{d} t \mathrm{~d} A_{n}
$$

where $U$ is the phase-averaged axial velocity, $T$ is the excitation period and $A_{n}$ is the cross-sectional area of the nozzle exit. This average velocity can be used to define a jet Reynolds number, given by

$$
R e_{\bar{U}}=\frac{\bar{U} d}{\nu}
$$

where $\nu$ is the kinematic viscosity of the working fluid and $d$ is the slot width or orifice diameter. An important length scale in synthetic jet flow is the non-dimensional stroke length, $L_{o} / d$, where

$$
L_{o}=\int_{0}^{T / 2} U_{\text {ave }}(t) \mathrm{d} t
$$


and $U_{\text {ave }}$ is the spatially averaged velocity. The stroke length represents the distance that the fluid travels during the expulsion portion of the cycle [8]. The excitation frequency $f$ can be non-dimensionalized as a Stokes number, $S$,

$$
S=\sqrt{\frac{2 \pi f d^{2}}{\nu}} .
$$

These non-dimensional parameters uniquely define the operating conditions of a synthetic jet and greatly influence its ability to transfer linear momentum. In particular, Holman et al. [10] showed that in order to achieve vortex escape after the expulsion stroke (defined as synthetic jet formation) $\operatorname{Re} / S^{2}>C$, where $C$ is a constant equal to 0.16 and 1 for axisymmetric and rectangular nozzles, respectively.

The average velocity that can be achieved by the jet is a function of three groups of parameters: geometry, fluid properties and driver properties. Holman et al. [10] identified the following important geometric properties: orifice diameter $d$ (or length and width for a $2 \mathrm{D}$ slot), nozzle length $L_{n}$, radius of curvature of the outer orifice edge, and the cavity volume $V_{c}$. The relevant properties of the driver are the oscillation frequency $f$, the fundamental resonant frequency $f_{d}$ and the volume displacement $\Delta V$. These parameters also affect the frequency response of the synthetic jet velocity [7, 5]. Absent from the geometric properties is the shape of the cavity. Typically, it is assumed that cavity shape is not important and can be designed without constraint, as has been suggested based on a numerical study from Utturkar et al. [26] and later by Jain et al. [11]. Utturkar et al. [26] used incompressible flow simulations to study the flow in rectangular cavities where the aspect ratio and location of the oscillating diaphragm(s) was varied. Cavities containing multiple oscillating diaphragms were also considered, where the oscillation amplitude was adjusted in order to maintain the same volume displacement in each case. The authors found that cavity shape and diaphragm placement had negligible impact on the actuator performance in both quiescent conditions or with cross-flow. However, the authors pointed out the inherent short-coming in using incompressible simulations as the flow inside a synthetic jet cavity is often compressible, and may exhibit higher sensitivity to the cavity design. Gallas [6] showed numerically and experimentally that the flow is incompressible for $f / f_{H}<0.5$, where $f_{H}$ is the Helmholtz frequency of the cavity. Synthetic jets are often operated at or near the Helmholtz frequency, therefore incompressible simulations are not sufficient to describe the flow environment. Jain et al. 
[11] employed compressible flow simulations in an axisymmetric synthetic jet and paid close attention to the modelling of the diaphragm motion. They briefly compared the standard cylindrical cavity to conical and parabolic shapes and found little difference in both the exit plane velocity and mass flow for the three shapes. For both Jain et al. [11] and Utturkar et al. [26], it is difficult to tell whether geometric similarity was maintained in the cavity comparisons since it was not explicitly stated if the cavity volume remained constant as the shape changed.

The goal of the present study is to provide the first experimental results concerning the influence of cavity shape on synthetic jet performance and determine whether exit plane velocity and momentum flux are insensitive to cavity shape. Three axisymmetric cavity shapes with equivalent geometric parameters are compared to a baseline cylindrical shape. The performance of the synthetic jet will be evaluated in the context of flow control applications, where jet momentum is the desired output quantity to be maximized. Unlike Utturkar et al. [26], where the goal was to investigate the diaphragm placement and cavity shape for practical design purposes, the present work focuses on jet performance improvement by modifying cavity shape.

\section{Experimental Setup}

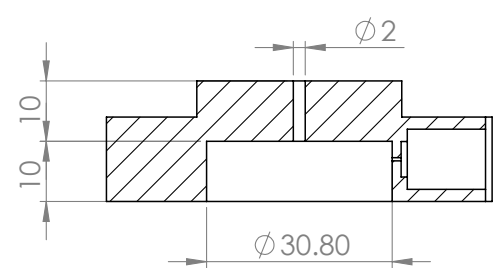

(a) Cylindrical (S1)

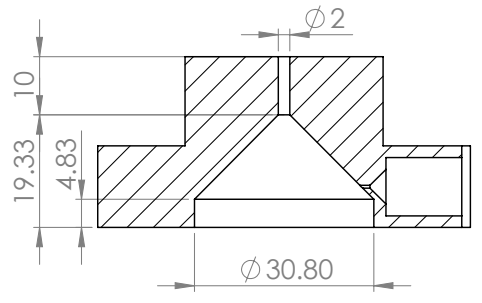

(b) Conical (S2)

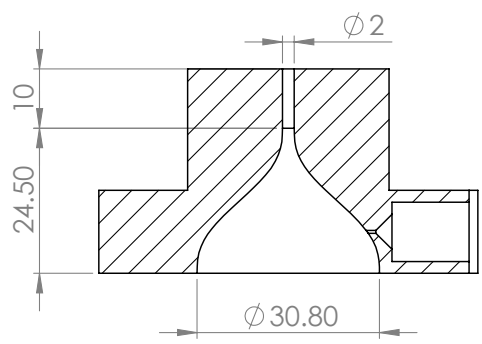

(c) Contraction (S3)

Figure 1: Constant volume axisymmetric synthetic jet cavity cross sections (dimensions in millimetres).

Three synthetic jet cavities using air as the working fluid were designed in order to study the effect of cavity shape on jet performance. A cylindrical cavity shape (Figure 1a) serves as the baseline case since it is most common for axisymmetric synthetic jets. Figures 1 (b) and (c) show the two other shapes selected 
for comparison: conical with a short cylindrical section, and an axissymmetric contraction defined by a 5 th order polynomial [2]. The cylindrical, conical and contraction cavity shapes will be referred to as S1, S2 and S3, respectively. It is assumed that geometries S2 and S3 may produce larger velocities at the nozzle exit than S1 due to a lower pressure loss during the expulsion portion of the cycle. The conical and contraction shaped cavities also reduce the dead zone present at the sharp corner inside the cylindrical cavity. To maintain geometric similarity between the three cavities, the following parameters were held constant: $d=2$ $\mathrm{mm}, L_{n}=10 \mathrm{~mm}$ and $V_{c}=9313 \mathrm{~mm}^{3}$. An orifice diameter of $2 \mathrm{~mm}$ was determined to be the minimum size where adequate spatial resolution could be achieved to allow velocity measurements across the orifice. The cavity diameter was held constant at the diameter of the driver $(30.8 \mathrm{~mm})$ and the cavity length was increased in order to maintain constant $V_{c}$. A Thunder TH-5C piezoelectric diaphragm manufactured by Face International Corporation was used to drive the synthetic jets. This piezoelectric diaphragm reaches a maximum centreline displacement of $0.127 \mathrm{~mm}$ when a voltage of $420 \mathrm{~V}$ (peak-to-peak) is applied. The input signal to the diaphragm is created using a Rigol DG1022 function generator and amplified by a Mide QPA3202 power amplifier. The diaphragm was rigidly clamped between two plates using eight bolts distributed evenly around its circumference. These plates were then secured to the desired jet cavity using another eight bolts and two dowel pins to ensure proper alignment. A torque wrench was used to apply $5.1 \pm 0.2 \mathrm{Nm}$ to the bolts each time a synthetic jet was assembled. All parts were CNC machined from aluminum. This design allowed a single piezoelectric diaphragm to be used for each cavity, thereby ensuring constant driver properties and boundary conditions (Figure 2).

Measurement of the cavity fluctuating pressure was performed using a GRAS 1/4" 40BP microphone with a dynamic range of $172 \mathrm{~dB}$ and $\pm 1 \mathrm{~dB}$ frequency response from $10 \mathrm{~Hz}-25 \mathrm{kHz}$. Due to the non-planar walls of the synthetic jet cavities, the microphone was mounted behind a pin hole with diameter $d_{m}=0.5$ mm. The pin hole length was $l_{m}=1.5 \mathrm{~mm}$ such that $l_{m} / d_{m} \geq 2$, as suggested by Tsuji et al. [25]. Furthermore, the effect of Helmholtz resonance on pressure measurements was removed by designing the gap between the microphone diaphragm and pinhole such that the resonance frequency was $3.8 \mathrm{kHz}$, well above the investigated frequency range. The microphone was operated without its protection grid and was held in place at the correct location using a close-fitting sleeve (Figure 2). 


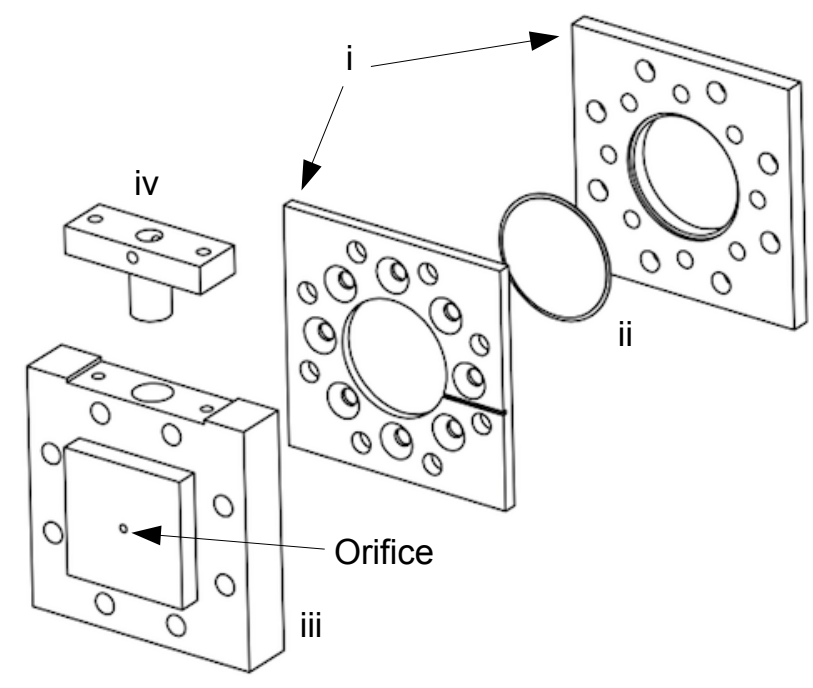

Figure 2: Synthetic jet actuator exploded assembly schematic. i. Diaphragm clamping plates. ii. Piezoelectirc diaphragm. iii. Cavity/nozzle plate. iv. Microphone sleeve.

The velocity at the orifice exit-plane was measured using hot-wire anemometry. A single-wire probe with wire diameter $d_{w}=2.5 \mu \mathrm{m}$ and prong spacing of approximately $2.5 \mathrm{~mm}$ was held parallel with the nozzle, as shown in Figure 3. The tungsten wire was plated with copper in order to produce an exposed sensing length of $l_{w}=0.5 \mathrm{~mm}$. The sensing length was confirmed under microscope. This wire geometry provides adequate spatial resolution while maintaining $l_{w} / d_{w} \geq 200$ [3]. A Dantec 56C01 main unit with 56S17 CTA bridges was used to measure the voltage from the hot-wire probe. Calibration was performed from $1.6-8 \mathrm{~m} / \mathrm{s}$ before each experiment using a Dantec 55D90 calibration unit and a King's Law fit to the data was employed in order to interpret velocities below the calibration range. Positioning of the hot-wire probe and alignment with the nozzle centreline was accomplished using manual traverses with fine adjustment. Radial profiles of velocity were measured by traversing the hot-wire probe across the orifice using a step-motor-driven traverse with a resolution of 800 steps $/ \mathrm{mm}$. The entire apparatus including synthetic jet and probe traverses were contained in a large plexiglass enclosure measuring approximately $1 \times 0.4 \times 0.4 \mathrm{~m}^{3}$. The measurement uncertainty due to calibration for all mean velocity quantities is approximately $3 \%$ based on the error propagation methodology of Yavuzkurt [28].

Data was sampled at $20 \mathrm{kHz}$ and $10^{5}$ points were acquired for each measurement. The function generator 


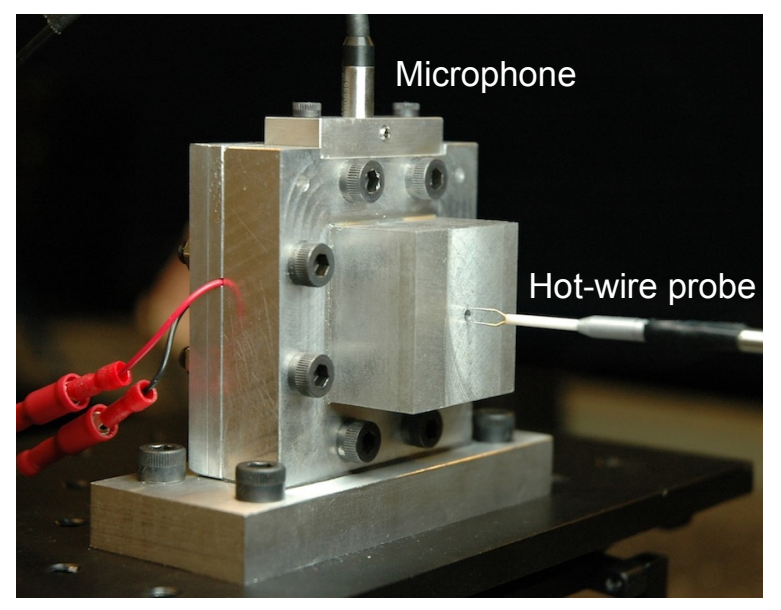

Figure 3: Experimental setup showing the synthetic jet assembly with contraction cavity installed.

voltage was acquired simultaneously with the microphone and hot-wire in order to provide a reference signal for phase averaging. Phase averaged velocity and pressure cycles were computed from 4848 individual cycles with a resolution in phase of $1.8^{\circ}$. Phase-averaged quantities were converged to within $1 \%$ at the $95 \%$ confidence interval over the entire cycle in all cases.

\section{Results}

\subsection{Synthetic jet characterization}

The frequency response of the centreline jet velocity at the exit plane, $U_{c l}$, and the cavity pressure, $p^{\prime}$, were characterized from $f=100-3000 \mathrm{~Hz}$ for peak-to-peak voltages $E=150,200$ and $250 \mathrm{~V}$. The excitation frequency was incremented by $50 \mathrm{~Hz}$ steps, except near sharp resonant peaks where additional measurements in $10 \mathrm{~Hz}$ increments were performed. Figure 4 shows a typical example of a phase-averaged velocity cycle for S1, $f=300 \mathrm{~Hz}$ and $E=250 \mathrm{~V}$, where $\theta$ is the phase angle relative to the input signal from the function generator. The area shaded in grey represents the extent of random data scatter for the entire data set. The lack of scatter in the data demonstrates the laminar behaviour of the jet at the exit plane and the repeatability of each cycle. Typical measurements of $U_{c l}$ and $p^{\prime}$ are shown in Figure 5 for the same conditions as Figure 4. The rectification of the jet velocity by the hot-wire was removed during post-processing. To de-rectify the signal, the larger peak in the jet velocity cycle was assumed to correspond to the expulsion 
portion of the cycle. This assumption is valid when the velocity is measured downstream of the exit plane, since the magnitude of the ingestion velocity decreases more rapidly moving away from the exit plane. Data near the local minima of the rectified cycle, where the jet velocity undergoes a change in direction, was omitted in the de-rectified velocity cycle and a spline fit was used to interpolate the regions between the remainder of the data. The markers on the velocity curve in Figure 5 identify the extent of the interpolated regions. The velocity and pressure each vary roughly sinusoidally, however the ingestion velocity does not have the same maximum magnitude as the expulsion. While this is partially accounted for by the fact that the hot-wire was located approximately $0.1 d$ downstream of the orifice, the maximum ingestion velocity at the centreline of the exit plane does not need to match the maximum expulsion velocity in order for the jet to have zero-net-mass-flux. Measurements of the radial profile of $U$ (presented subsequently) show that during both the ingestion and expulsion halves of the cycle, the radial velocity profile can differ substantially from the idealized top-hat shape.

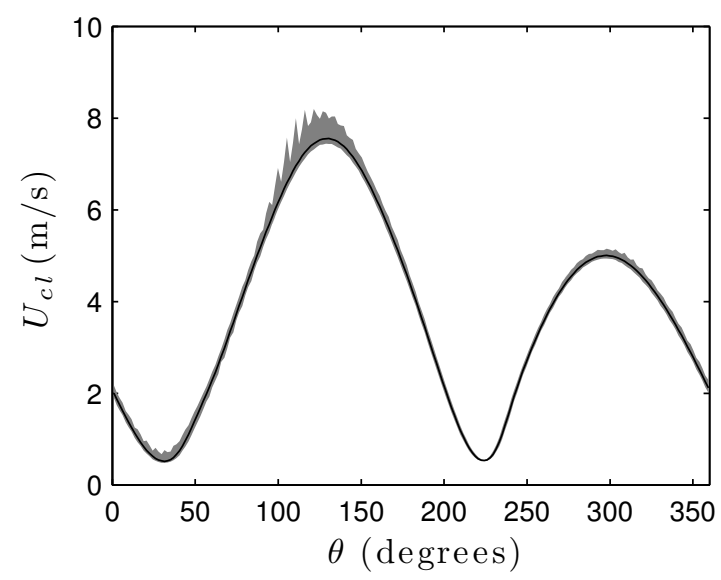

Figure 4: Phase-averaged centerline jet velocity for S1, $f=300 \mathrm{~Hz}$ and $E=250 \mathrm{~V}$. Shaded grey region shows the extent of random data scatter.

Figures 6 and 7 show the magnitude response of $U_{c l}$ and $p^{\prime}$, respectively, to sinusoidal excitation. The quantity plotted is the maximum value in the phase averaged cycle, since this value is more accurate than the rms value for low velocities. Based on the lumped-element model of Gallas et al. [7] relating the jet velocity to input voltage, two resonant frequencies $\left(f_{1,2}\right)$ are expected for a piezoelectrically driven synthetic 


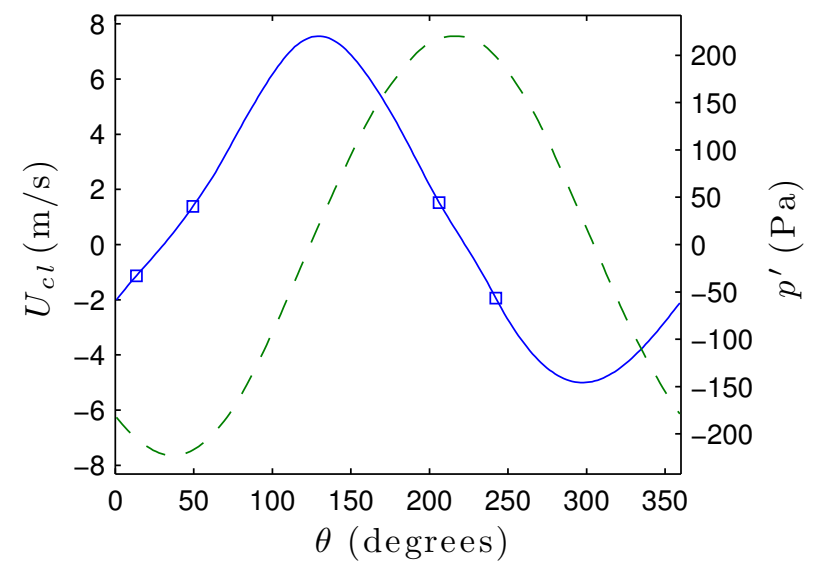

Figure 5: Centerline jet velocity (-) and pressure (- -) for S1, $f=300 \mathrm{~Hz}$ and $E=250 \mathrm{~V}$. The square markers indicate the extent of the interpolated regions.

jet. These values are bounded by, but not equal to, the Helmholtz frequency of the cavity $\left(f_{H}\right)$ and the natural frequency of the diaphragm $\left(f_{d}\right)$. For an axisymmetric orifice, the Helmholtz frequency is given by

$$
f_{H}=\frac{1}{2 \pi} \sqrt{\frac{3}{4} \frac{A_{n} c^{2}}{L_{n} V_{c}}},
$$

where $c$ is the speed of sound in the cavity [6]. As shown in Figure 6, two resonant frequencies are apparent for each cavity shape, with the lower resonant peak having significantly larger amplitude than the higher resonant peak. For the $\mathrm{S} 1$ and $\mathrm{S} 2$ cavities, $f_{1} \approx 300 \mathrm{~Hz}$, while $\mathrm{S} 3$ cavity has a lower peak at $f_{1} \approx 250 \mathrm{~Hz}$. Equation (5) finds $f_{H}=278 \mathrm{~Hz}$ for all three cavities, therefore the low frequency peak can be associated with Helmholtz resonance of the cavity. The Helmholtz frequency of each cavity was also measured experimentally by exciting the synthetic jet cavity (with no voltage applied to the piezoelectric diaphragm) using a loudspeaker placed in front of the orifice and driven by a function generator. Helmholtz resonance was identified as the frequency causing the largest value of $p^{\prime}[4,17]$. That frequency for both S1 and S2 was $f_{H, \exp }=280 \pm 1 \mathrm{~Hz}$ and for S3, $f_{H, e x p}=243 \pm 1 \mathrm{~Hz}$. The difference in $f_{H}$ for S3 can be explained by examining the variables in (5). These variables are well defined for S1 and S2, while the nozzle length $L_{n}$ is somewhat ambiguous for S3 due to the smooth transition between the nozzle and cavity. It is reasonable to assume that the "effective" nozzle length for S3 is larger, therefore causing a lower $f_{H}$. This explains the difference in $f_{1}$ for the three cavity 
shapes.

The second resonant peak shifts from $f_{2} \approx 2650 \mathrm{~Hz}$ at $E=150 \mathrm{~V}$ to $f_{2} \approx 2350 \mathrm{~Hz}$ at $E=250 \mathrm{~V}$ for each cavity. Since the $f_{1}$ peak is constant within measurement uncertainty $( \pm 5 \mathrm{~Hz})$, this suggests that $f_{d}$ is not constant with input amplitude and the piezoelectric diaphragm is behaving with some degree of non-linearity. Near Helmholtz resonance, $U_{c l}$ is approximately equal for the there cavities (albeit at lower frequencies for S3), while at higher frequencies $U_{c l, S 1}>U_{c l, S 2}>U_{c l, S 3}$. However, for frequencies above $\sim 450 \mathrm{~Hz}$, the jet velocity is very small, therefore frequencies near $f_{1}$ will be the focus of the remaining discussion.
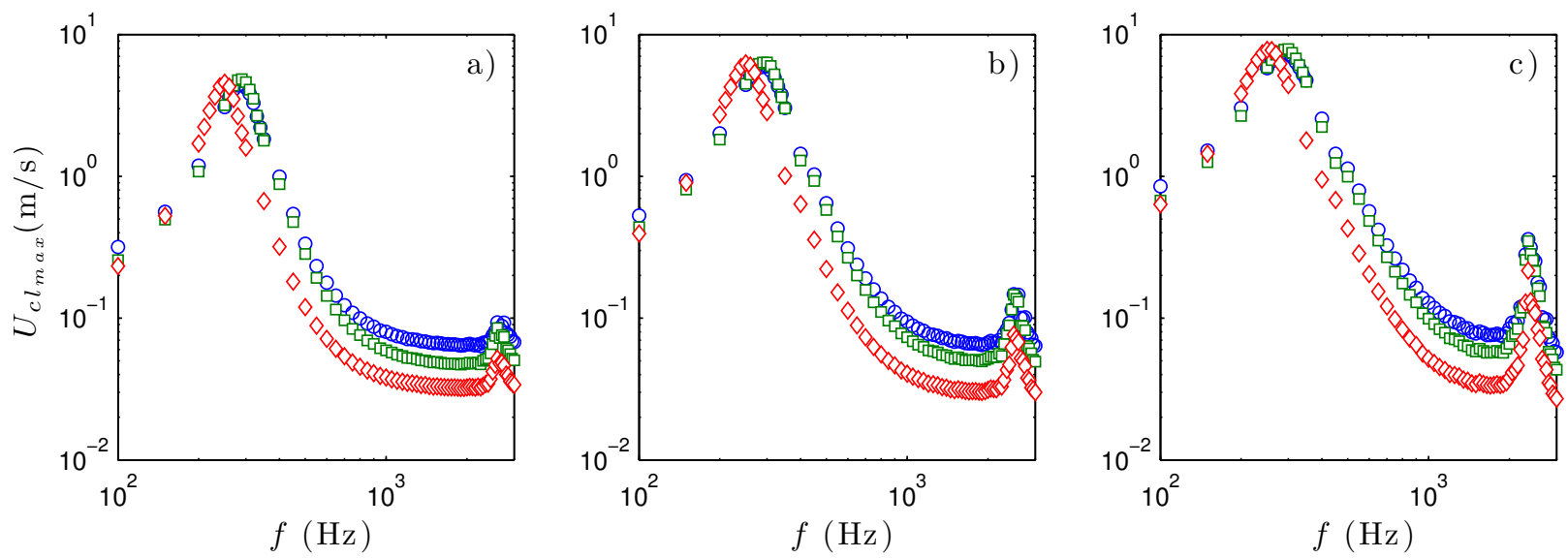

Figure 6: Maximum centreline jet velocity measured at the exit plane for a) $E=150 \mathrm{~V}, \mathrm{~b}) E=200 \mathrm{~V}$ and c) $E=250$ V. Markers indicate (o) S1, $(\square) \mathrm{S} 2$ and $(\diamond) \mathrm{S} 3$.

Several authors $[9,22,20]$ have employed a pressure-velocity synthetic jet characterization using the cavity pressure in place of a voltage-velocity characterization. The input voltage amplitude is incapable of accounting for factors such as actuator degradation and other external influences. Furthermore, voltage amplitude is not adequate when comparing synthetic jets which may have different geometries, drivers and Helmholtz frequencies, among other differences. A characterization based on cavity pressure is a suitable alternative since it is relatively easily acquired and is independent of the driver properties. Figure 7 highlights the issues involved in comparing different synthetic jets based on input voltage amplitude and frequency. Since S1 and S2 have identical $f_{H}$, there is very little difference in $p^{\prime}$ for these cavities, however this is not true for S3. Near the peak associated with Helmholtz resonance, the pressure versus frequency curve for S3 is shifted to the left 
due to a lower Helmholtz frequency. When the three cavities are operated at the same voltage amplitude and frequency in this range, the pressure may vary significantly. For example, at $f=200 \mathrm{~Hz}$ and $E=200 \mathrm{~V}, p^{\prime}$ is $66 \%$ larger for S3 than S1 or S2. The peak of $p^{\prime}$ at $f_{1}$ is noticeably larger for S3 than S1 or S2. Given that the flow through the synthetic jet nozzle is driven by the cavity pressure, the performance of different synthetic jets cannot be directly compared if cavity pressure differs. Therefore, comparing synthetic jets using cavity pressure rather than voltage amplitude is more appropriate. Persoons [19] developed a reduced-order model using a lumped element approach to relate the average velocity at the nozzle exit to the cavity pressure. Given the cavity pressure, geometric and fluidic properties of the synthetic jet, $\bar{U}$ can be estimated by

$$
\bar{U}=\frac{\overline{p^{\prime}}}{\rho c} \sqrt{\frac{2 V_{c}}{A_{n} L_{n}^{\prime}}}\left[\sqrt{\left(\frac{f}{f_{H}}\right)^{2}+\sqrt{\left(\frac{f}{f_{H}}\right)^{4}+K^{2}\left(\frac{V_{c} \bar{p}^{\prime}}{A_{n} L_{n}^{\prime} \rho c^{2}}\right)^{2}}}\right]^{-1},
$$

where $L_{n}{ }^{\prime}=L_{n}+2 \beta \sqrt{4 A_{n} / \pi}, \beta$ is an added mass coefficient for the nozzle and $K$ is a non-linear damping loss coefficient. This model is valid up to a frequency dependant limit $f<c / 16 L_{\max }$, where $L_{\max }$ is the largest linear dimension in the cavity or nozzle. From (6), the synthetic jet cavities in this study will operate under the same conditions when $f / f_{H}$ and $\overline{p^{\prime}}$ are constant. Any variation in the jet performance may be attributed to a lower loss coefficient $K$ or mass coefficient $\beta$.
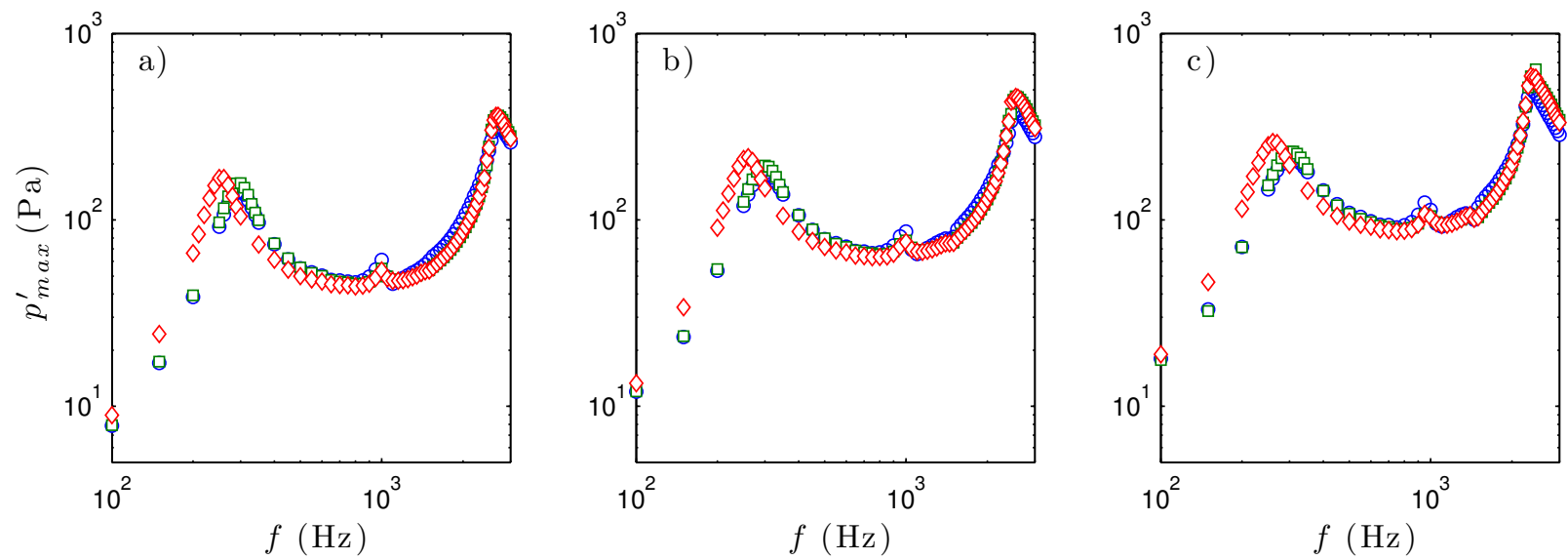

Figure 7: Cavity pressure for a) $E=150 \mathrm{~V}$, b) $E=200 \mathrm{~V}$ and c) $E=250 \mathrm{~V}$. Markers indicate (o) S1, ( $\square$ ) S2 and $(\diamond)$ S3. 


\subsection{Radial profiles of axial jet velocity}

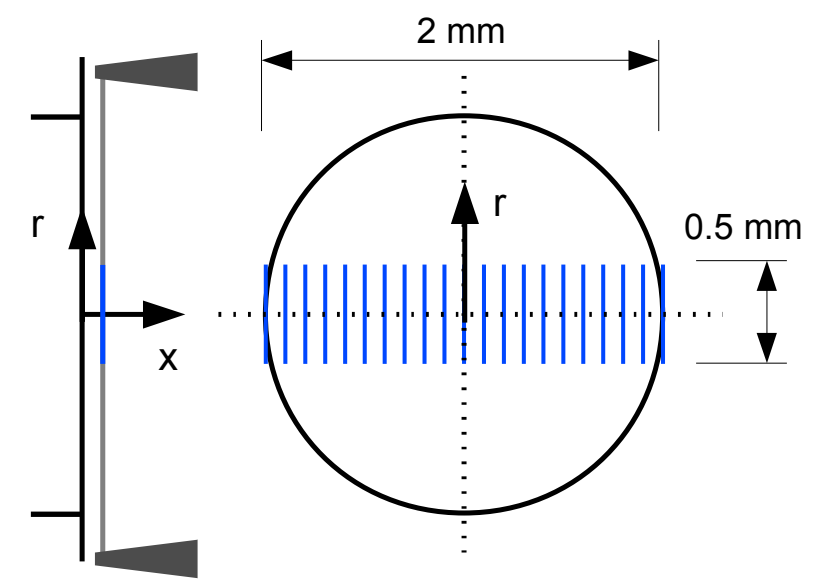

Figure 8: Scale drawing showing the radial hot-wire measurement locations across the orifice (wire diameter is emphasized so that it can be visualized). Solid vertical lines indicate the sensing length of the hot-wire probe. The origin of the cylindrical coordinate system is located at the centre of the orifice exit plane.

Radial profiles of $U$ were measured at $x / d=0.075$ by traversing the hot-wire from $r / d=-0.5$ to 0.5 in increments of $0.1 \mathrm{~mm}(0.05 \mathrm{~d})$. Figure 8 shows a scale drawing of the measurement stations with the hot-wire sensing length indicated. It is important to note that the prong spacing of the hot-wire probe was larger than the orifice diameter such that the prongs did not affect the jet. The centreline, $r=0$, was identified after each new cavity was installed by measuring the location where symmetry of the velocity profile was achieved. Three test cases were considered: 1) $f / f_{H}=0.71$ and $\left.\overline{p^{\prime}}=50 \mathrm{~Pa}, 2\right) f / f_{H}=1.07$ and $\overline{p^{\prime}}=160$ $\mathrm{Pa}$, and 3) $f / f_{H}=1.25$ and $\overline{p^{\prime}}=100 \mathrm{~Pa}$ (Table 1 ). Note that $f_{H}$ was taken as the experimentally measured value for each cavity shape. For a given frequency, the cavity pressure was adjusted by varying the voltage amplitude until the desired value was obtained.

Figure 9 shows the radial profiles of $U$ normalized by the largest centreline velocity at six phase locations for $f / f_{h}=0.71$ and $\overline{p^{\prime}}=50 \mathrm{~Pa}$ (Case 1). The phase angle $\phi$ is defined such that $\phi=90^{\circ}$ corresponds to maximum expulsion velocity. The results demonstrate that over the entire cycle, the velocity profiles have approximately equal shape for each cavity. However, the magnitude of the velocity is generally largest for S1 and slightly lower for S2, with S3 having the lowest velocities. Similar results are obtained for test Cases 2 

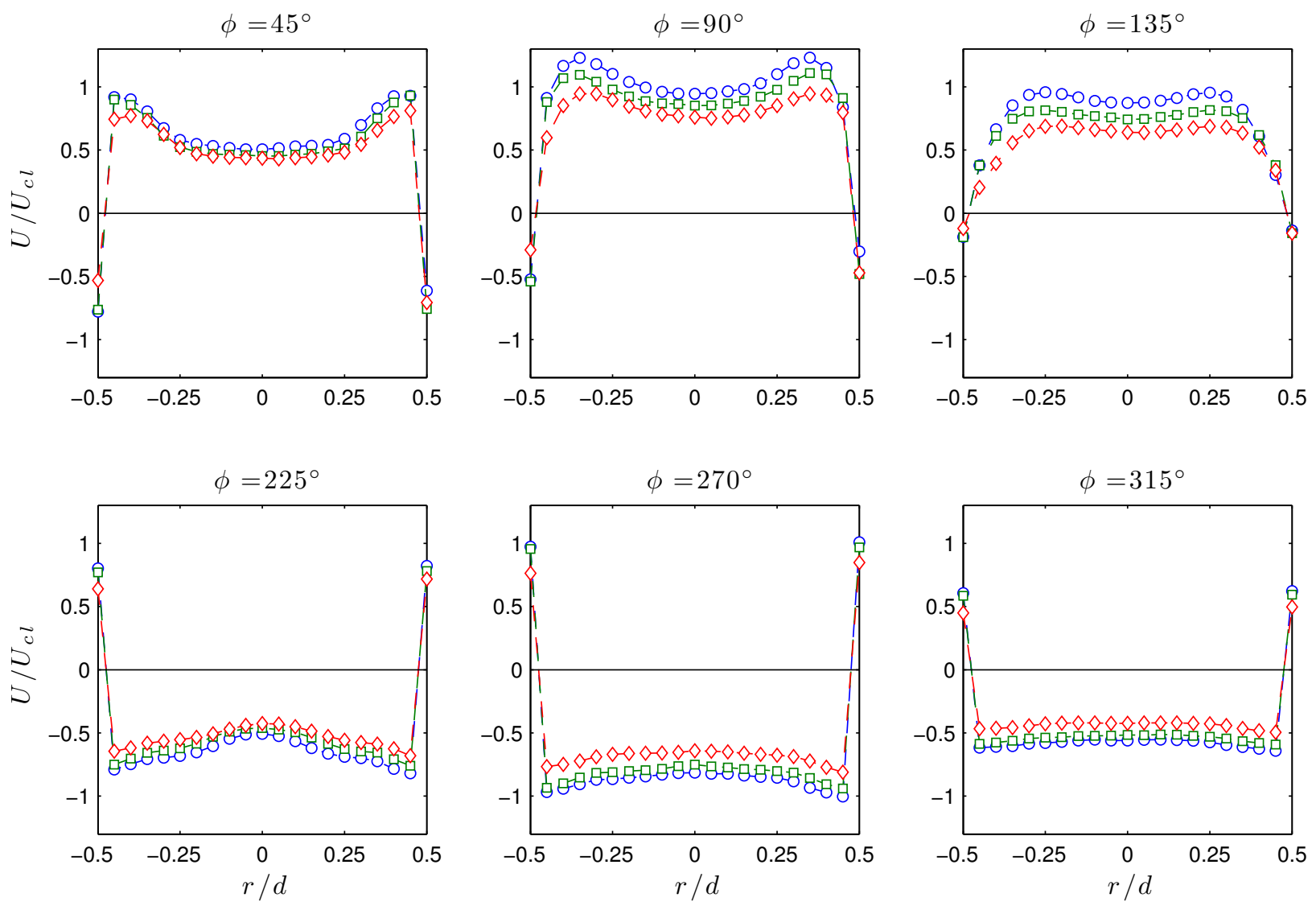

Figure 9: Radial profile of jet velocity at the exit plane for Case 1. Normalization is by the maximum centreline velocity. Markers indicate (o) S1, $(\square)$ S2 and $(\diamond)$ S3.

and 3, as shown in Figures 10 and 11, respectively. During the ingestion half of the cycle, the velocity profile for Cases 1 and 2 exhibits a top-hat shape that is expected of a sink flow. For Case 3, the ingestion velocity profiles deviate significantly from the top-hat shape. Large positive velocities up to $U_{j} / U_{c l}=1$ are also observed near $r / d= \pm 0.5$ during ingestion. Since the hot-wire probe senses the total velocity vector and is unable to distinguish flow direction, the radial velocity component that occurs near the orifice circumference during ingestion is likely the dominant component of the total velocity measured by the hot- wire. During the positive-going half of the expulsion stroke $\left(0<\phi \leq 90^{\circ}\right)$, negative velocity is observed near the orifice circumference due to vortex formation. This was also observed by Shuster and Smith $[21]$ at $x / d=0.1$ using PIV measurements. However, the magnitude of these velocities in the present experiment is exaggerated due 
to the radial velocity component and, to a lesser extent, radiative heating of the hot-wire with the orifice surface. Because of this, there is significant uncertainty in the measurement of velocity at $r / d= \pm 0.5$. The velocity data at $r / d= \pm 0.5$ was omitted from the calculation of the mean velocity, flow rate and momentum flux, which are presented subsequently. The expulsion portion of the cycle is characterized by a region of high-velocity near the wall and an inviscid core. This velocity profile is typical of fully developed flow in a circular duct that is driven by an oscillating pressure gradient and is characterized by the Stokes number [27]. For $S<2$, the velocity profile resembles a quasi-steady Poiseuille flow where the ratio of the spatially averaged velocity to the centreline velocity is 0.5 . As $S$ increases to approximately 20 , the average velocity reaches $90 \%$ of the centreline velocity and continutes to asymptote towards $100 \%$ as $S \rightarrow \infty$. The Stokes numbers for Cases 1 through 3 are approximately 18, 22 and 25, respectively (Table 1). Based on $S$ and the relatively large nozzle length to orifice diameter, $L_{n} / d=5$, fully developed oscillatory flow profiles are not unexpected. The results show further agreement with theory in that the extent of the inviscid core during expulsion increases with Stokes number [27], as shown in Figures 10 and 11. Figure 11 demonstrates that the Stokes number also influences the shape of the velocity profile during ingestion, which resembles an inverse parabola for $S \approx 25$. 

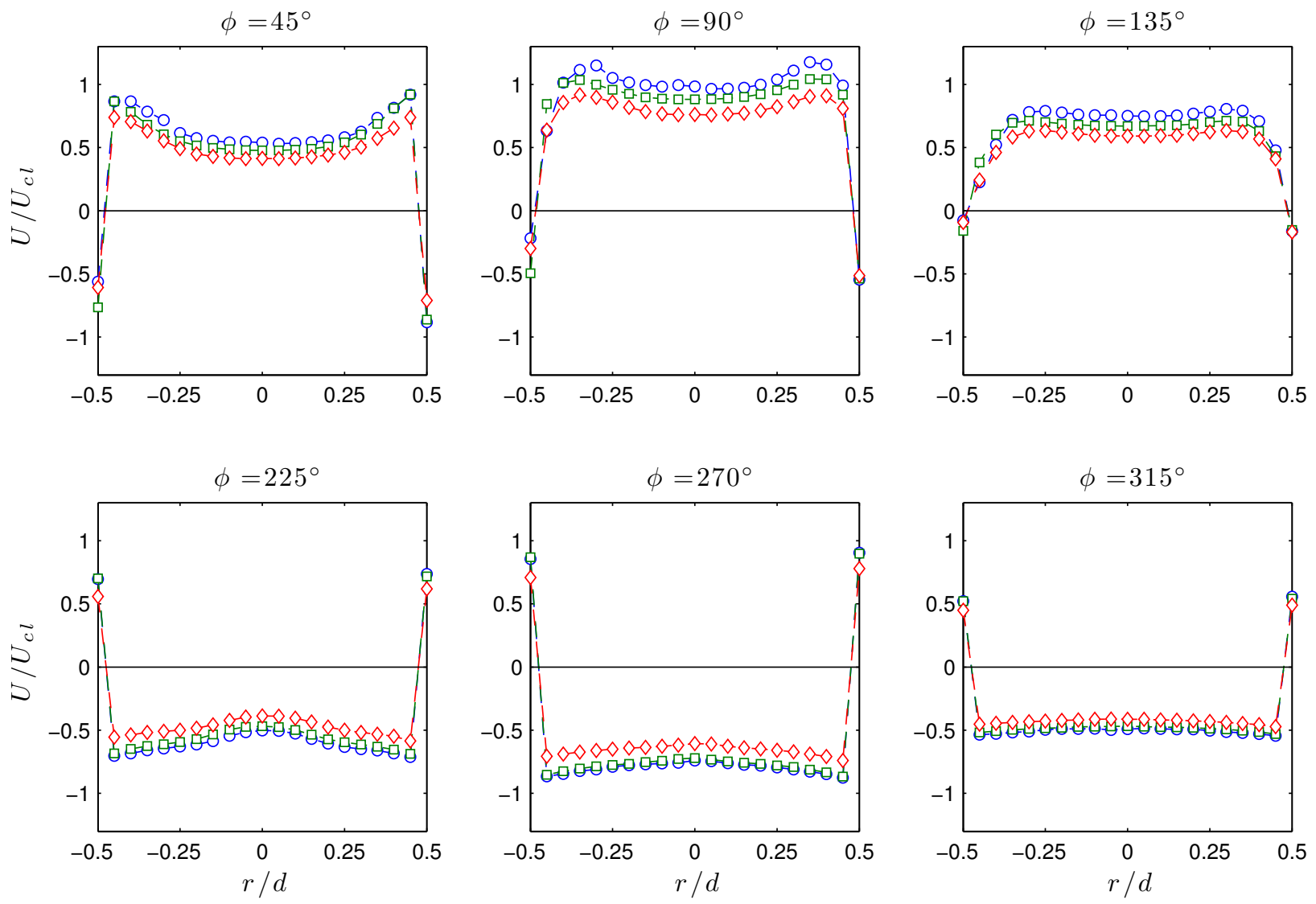

Figure 10: Radial profile of jet velocity at the exit plane for Case 2. Normalization is by the maximum centreline velocity. Markers indicate $(\circ) \mathrm{S} 1,(\square) \mathrm{S} 2$ and $(\diamond) \mathrm{S} 3$. 

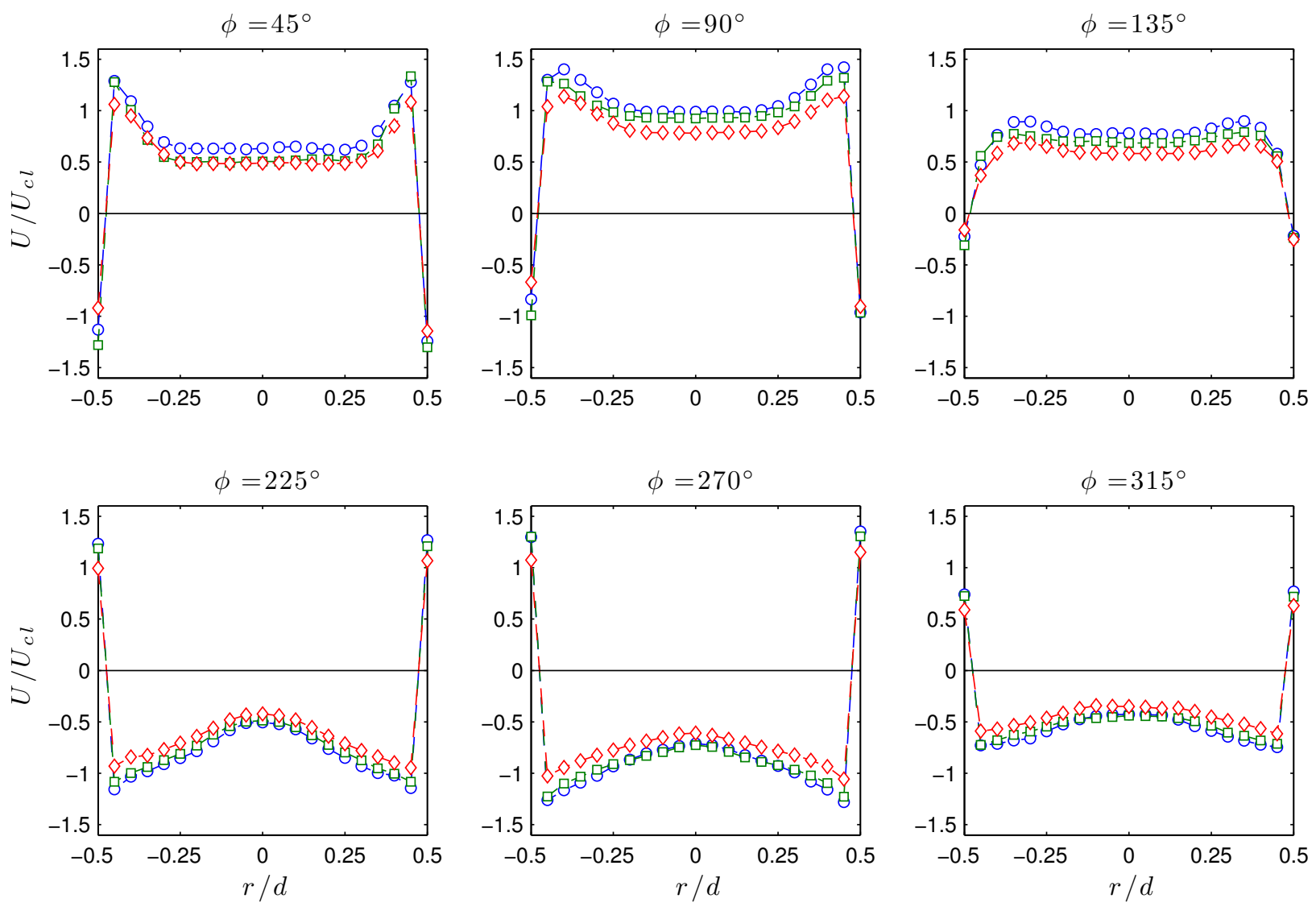

Figure 11: Radial profile of jet velocity at the exit plane for Case 3. Normalization is by the maximum centreline velocity. Markers indicate $(\circ) \mathrm{S} 1,(\square) \mathrm{S} 2$ and $(\diamond) \mathrm{S} 3$.

Table 1 summarizes the jet performance parameters for each cavity shape. For each of the test cases, $R e_{\bar{U}}$ varies within $\pm 10 \%$ of the mean, while $R e_{\bar{U}} / S^{2}$ and $L_{o} / d$ show much closer agreement. Consistent with the velocity profiles in Figures 9 to 11, the Reynolds number increases from cavity shape S3 to S1 (i.e., $\left.R e_{\bar{U}, S 1}>R e_{\bar{U}, S 2}>R e_{\bar{U}, S 3}\right)$. The Reynolds number and jet formation criterion suggest that a laminar jet is formed in all cases. The mean dimensionless stroke length varies from $L_{o} / d=1.2$ for Case 3 to $L_{o} / d=4.2$ for Case 2.

The definition of a synthetic jet requires that at $x / d=0$, the temporally averaged flow rate over a cycle is zero. The flow rate through a circular orifice is given by 
Table 1: Test case parameters and synthetic jet performance results for radial profile measurements of $U$. Constant $\overline{p^{\prime}}$ is achieved at a given frequency by adjusting the voltage amplitude. $\operatorname{Re} \bar{U} / S^{2}>0.16$ indicates that a jet is formed [10]. Deviation in zero-net-mass-flux is quantified by the cycle averaged flow rate, where positive values of $Q_{\text {ave }} / Q_{\mathrm{rms}}$ indicate excess flow rate in the expulsion stoke

\begin{tabular}{|c|c|c|c|c|c|c|c|c|}
\hline Case \# & $f / f_{H}$ & $\overline{p^{\prime}}(\mathrm{Pa})$ & Shape & $R e_{\bar{U}}$ & $S$ & $R e_{\bar{U}} / S^{2}$ & $L_{o} / d$ & $\begin{array}{l}Q_{\text {ave }} / Q_{\mathrm{rms}} \\
(\%)\end{array}$ \\
\hline \multirow[t]{3}{*}{1} & 0.71 & 50 & S1 & 287 & 18 & 0.90 & 2.8 & 7 \\
\hline & & & $\mathrm{S} 2$ & 263 & 18 & 0.82 & 2.6 & 7 \\
\hline & & & S3 & 229 & 17 & 0.82 & 2.6 & 7 \\
\hline \multirow[t]{3}{*}{2} & 1.07 & 160 & $\mathrm{~S} 1$ & 676 & 22 & 1.41 & 4.5 & 11 \\
\hline & & & $\mathrm{S} 2$ & 616 & 22 & 1.28 & 4.1 & 8 \\
\hline & & & S3 & 538 & 20 & 1.29 & 4.1 & 9 \\
\hline \multirow[t]{3}{*}{3} & 1.25 & 100 & S1 & 258 & 25 & 0.40 & 1.3 & 4 \\
\hline & & & $\mathrm{S} 2$ & 235 & 25 & 0.37 & 1.2 & 2 \\
\hline & & & S3 & 204 & 24 & 0.37 & 1.2 & 2 \\
\hline
\end{tabular}

$$
Q(t)=\int_{0}^{d / 2} 2 \pi r U(r, t) \mathrm{d} r
$$

The velocity profiles in Figures 9 through 11 show that a non-zero net mass flux may exist at the measurement plane $x / d=0.075$. Nani and Smith [15] showed that if the flow rate is computed at $x / d>0$ using only a plane parallel to the orifice face, the flow rate during the ingestion phase may be considerably under-predicted due to significant flow in the radial direction. However, their results demonstrated that at $x / d=0.0726$, the flow in the radial direction was small relative to the axial flow. Typical examples of the flow rate over a phase averaged cycle are shown in Figure 12 for S2 (Case 2) and S3 (Case 3). Each curve is normalized by its respective maximum. Also included in this plot are horizontal lines indicating the integrated value $Q_{\text {ave }}$ over the cycle. The mean value of $\mathrm{Q}$ is nearly zero for Case 3, while for Case 2 there is excess flow rate in the expulsion portion of the cycle. For all the cases considered, the mean flow rate was no more than $11 \%$ 
of the rms value, suggesting that the measurements are sufficiently close to $x / d=0$ to be representative of the flow at the exit plane. Furthermore, this provides confidence that the velocity measured by the hot-wire at $-0.45 \leq r / d \leq 0.45$ has been correctly interpreted as axial velocity. The small excess flow rate in the expulsion stroke, positive $Q_{\text {ave }}$, provides a measure of the radial flow that was not accounted for. Table 1 summarizes the deviation from zero-net-mass-flux according to $Q_{\text {ave }} / Q_{\mathrm{rms}}$.

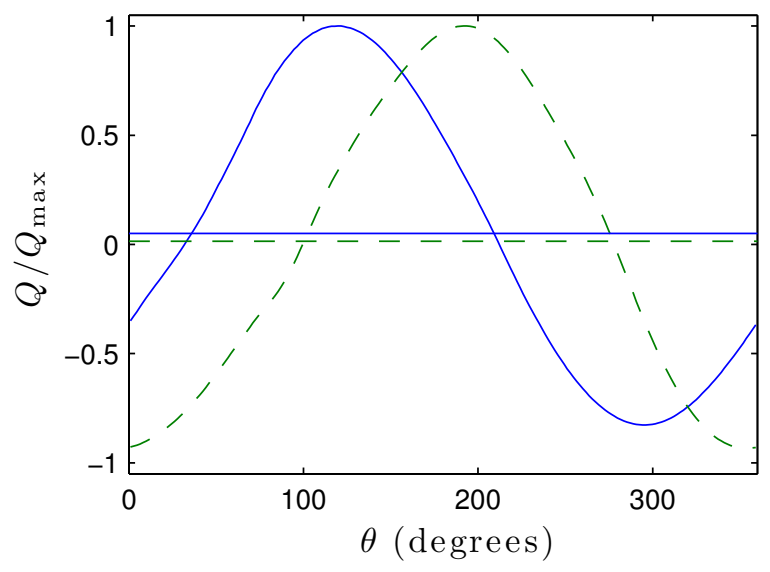

Figure 12: Phase averaged flow rate for $(-) \mathrm{S} 2$, Case $2\left(Q_{\max }=2.5 \times 10^{-5} \mathrm{~m}^{3} / \mathrm{s}\right)$ and (- $)$ S3, Case 3 $\left(Q_{\max }=8.2 \times 10^{-6} \mathrm{~m}^{3} / \mathrm{s}\right)$ normalized by their respective maxima. Horizontal lines show the mean value obtained by integrating over the entire cycle.

\subsection{Jet momentum}

In flow control applications, the purpose of a synthetic jet actuator is to impart momentum on the boundary layer through the formation of a vortex pair or ring. Therefore, the performance of the three cavity shapes can be further compared by considering the time-averaged momentum flux, calculated as $[23,16]$

$$
J=\rho \int_{0}^{\infty} 2 \pi r\left(U^{2}+{\overline{u^{\prime}}}^{2}\right) \mathrm{d} r
$$

where $u^{\prime}$ is the fluctuating axial velocity. At $x / d=0.075$, integration was computed over both $0 \leq r / d \leq 0.45$ and $-0.45 \leq r / d \leq 0$ and the momentum flux was taken as the mean value. The average measurement uncertainty in $J$ is $\pm 5 \%$. Figure 13 shows the effect of cavity shape on $J$ for each test case. The results 
demonstrate that S1 achieves the largest momentum in each case and the momentum decreases sequentially from S1 to S3. The momentum flux of S1 is approximately $15 \%$ and $36 \%$ larger than that of $\mathrm{S} 2$ and $\mathrm{S} 3$, respectively.

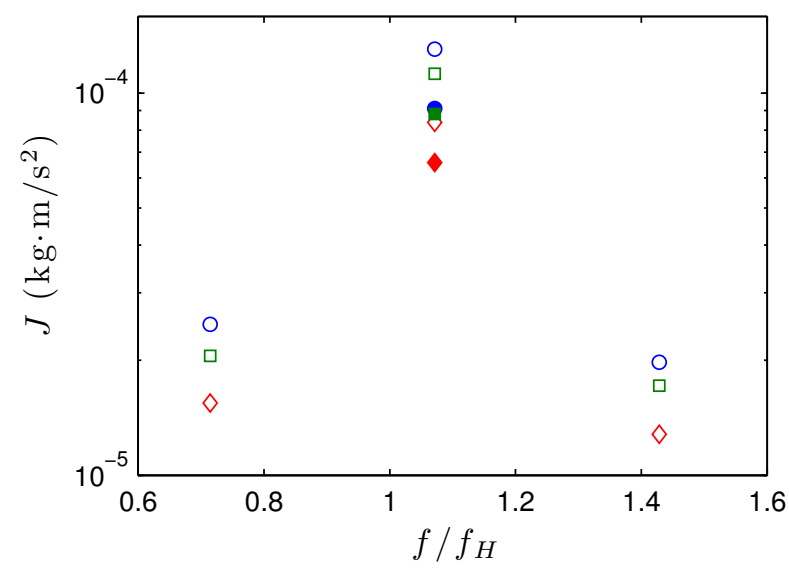

Figure 13: Mean jet momentum flux for each test case. Markers indicate (o) S1, ( $\square$ ) S2 and ( $\diamond)$ S3. Open symbols are at $x / d=0.075$ and filled symbols are at $x / d=5$.

The momentum flux for Case 2 was also measured at a location downstream of the orifice. A streamwise location of $x / d=5$ was selected since it was beyond the region where the vortex ring was formed and the flow was not affected by the ingestion stroke at this location. It was also assumed that the radial velocity was small relative to the axial velocity at $x / d=5$. Since Cases 1 and 3 have considerably lower velocity than Case 2, these test cases were not included in the measurements. Figure 14 shows the time-averaged profiles of $U$ normalized by the maximum centerline velocity at $x / d=5$. As expected, the time-averaged velocity profiles resemble that of a conventional round jet that is developing. Interestingly, it would appear that the jet produced by S1 has experienced more rapid decay than S2 since the velocity profiles have nearly equal magnitude at this location. This effect is reflected in the momentum flux, shown by the filled symbols in Figure 13. The momentum flux of S1 and S2 differ by less than 10\%, while that of S3 is approximately $30 \%$ smaller. These results also demonstrate that unlike a conventional jet, the momentum decreases downstream of the orifice. This behaviour is typical for synthetic jets and is caused by the existence of an adverse streamwise pressure gradient near the jet orifice as a result of the suction stroke [23, 24]. 


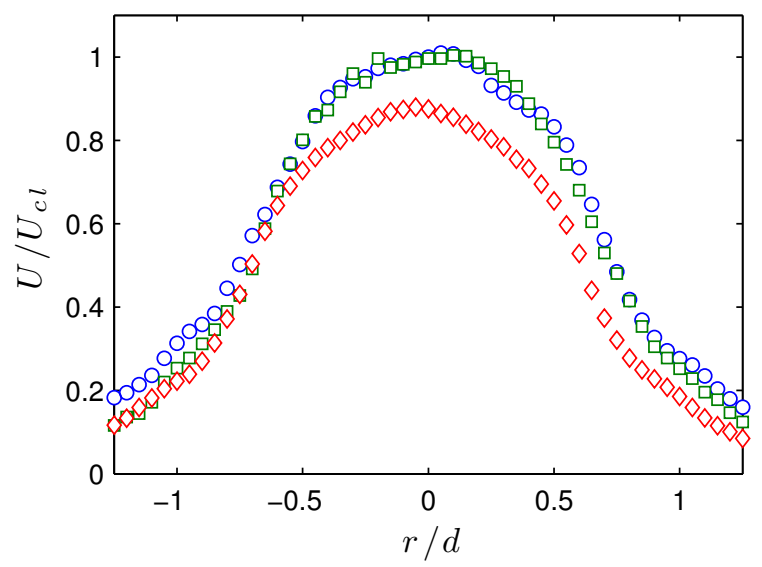

Figure 14: Cross-stream distribution of the time-averaged axial velocity at $x / d=5$ for Case 2 . Normalization is by the maximum centreline velocity. Markers indicate (o) S1, ( $\square$ ) S2 and $(\diamond)$ S3.

These results are certainly surprising since it was hypothesized that if cavity shape were to have any effect on the jet momentum, the opposite trend would be observed and S3 would achieve the largest momentum flux. If the tested cavity shapes were used as steady jets (i.e., continuous blowing), this would be the case due to a decreasing pressure drop from S1 to S2 to S3. The fact that S1 achieves the highest momentum flux highlights the complexity of the oscillatory flow and may be due to interaction within the cavity with the local recirculating flow. At the beginning of the suction stroke, vorticity is generated at the sharp outer edge of the orifice. These vortices are then ingested into the cavity (e.g., Kotapati et al. [13]). During expulsion, the flow attempting to leave the cavity will be in counterflow with the decelerating vortices from the ingestion stroke. For the baseline cylindrical cavity, there is sufficient room in the cavity for the outgoing flow to move around these vortices. It is possible that as the region of the cavity near the neck becomes narrower for S2 and S3, the outgoing flow effectively encounters a larger blockage from the suction flow. Figure 15 shows a schematic of the expected flow behaviour.

\subsection{Efficiency considerations}

The efficiency of a synthetic jet actuator can be defined in a number of different ways based on its intended purpose. As mentioned above, the output quantity of interest in aerodynamic flow control is jet momentum. 


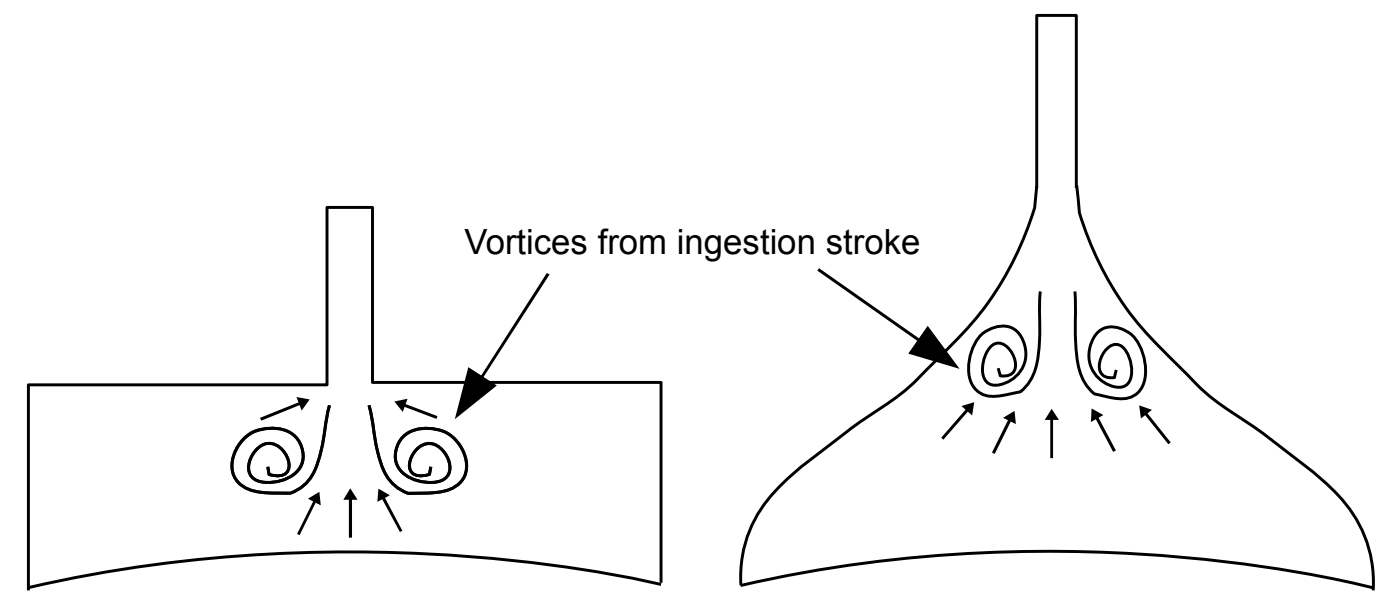

Figure 15: Theorized cavity flow details during the expulsion stroke for cavities S1 (left) and S3 (right).

Persoons [19] defined a fluidic efficiency based on jet momentum, viz.

$$
\eta=\frac{\rho A_{n} \bar{U}^{2} c}{E_{\mathrm{rms}} I_{\mathrm{rms}} \cos \Phi}
$$

where $E_{\mathrm{rms}}$ and $I_{\mathrm{rms}}$ are the rms voltage and current supplied to the piezoelectric diaphragm, respectively, and $\Phi$ is the phase angle between voltage and current. The real power consumed by the actuator is defined as $P=E_{\mathrm{rms}} I_{\mathrm{rms}} \cos \Phi$. Due to the capacitive nature of the piezoelectric diaphragm driving the synthetic jet, the power used by the actuator (real power) is small compared to the reactive power that is delivered back to the supply. While Persoons [19] introduced this measure of fluidic efficiency based on jet momentum, the author did not present results for this parameter. Figure 16 shows the efficiency plotted against $f / f_{H}$ for each cavity. Maximum efficiency is achieved at the resonant peak $f_{1}=1.07 f_{H}$ for all geometries. At $f / f_{H}=0.71$ and 1.43 , the efficiency is approximately equal for the three cavity shapes. However, at $f / f_{H}=1.07$ the efficiency of S3 is approximately $20 \%$ larger than that of S1 and S2. As shown in Table 1, the variation in $R e_{\bar{U}}$ within each test case is small and increases sequentially from S3 to S2 to S1, therefore the large difference in $\eta$ is due to the power consumed by the actuator. The electrical power consumption is also shown in Figure 16. Due to the difference in Helmholtz frequencies, $f / f_{H}=1.07$ corresponds to $f=260 \mathrm{~Hz}$ for $\mathrm{S} 3$ and $f=300 \mathrm{~Hz}$ for $\mathrm{S} 1 / \mathrm{S} 2$. Piezoelectric diaphragms behave essentially as a capacitor such that their reactance is inversely proportional to frequency. This implies that for a given excitation voltage and $f / f_{H}$, S3 will draw 
less current than S1 and S2. Furthermore, the voltage amplitude required to match the cavity pressure was not necessarily equal for a given $f / f_{H}$, as shown in Figure 17 . There is substantial variation in $\overline{p^{\prime}}$ near the resonant peak from approximately $f / f_{H}=0.9$ to 1.25 , with S3 having the largest values. This suggests that near Helmholtz resonance, the shape of the cavity influences the maximum pressure that can be achieved for a given voltage amplitude. It is therefore not surprising that $\eta$ primarily follows the trend of $P^{-1}$.
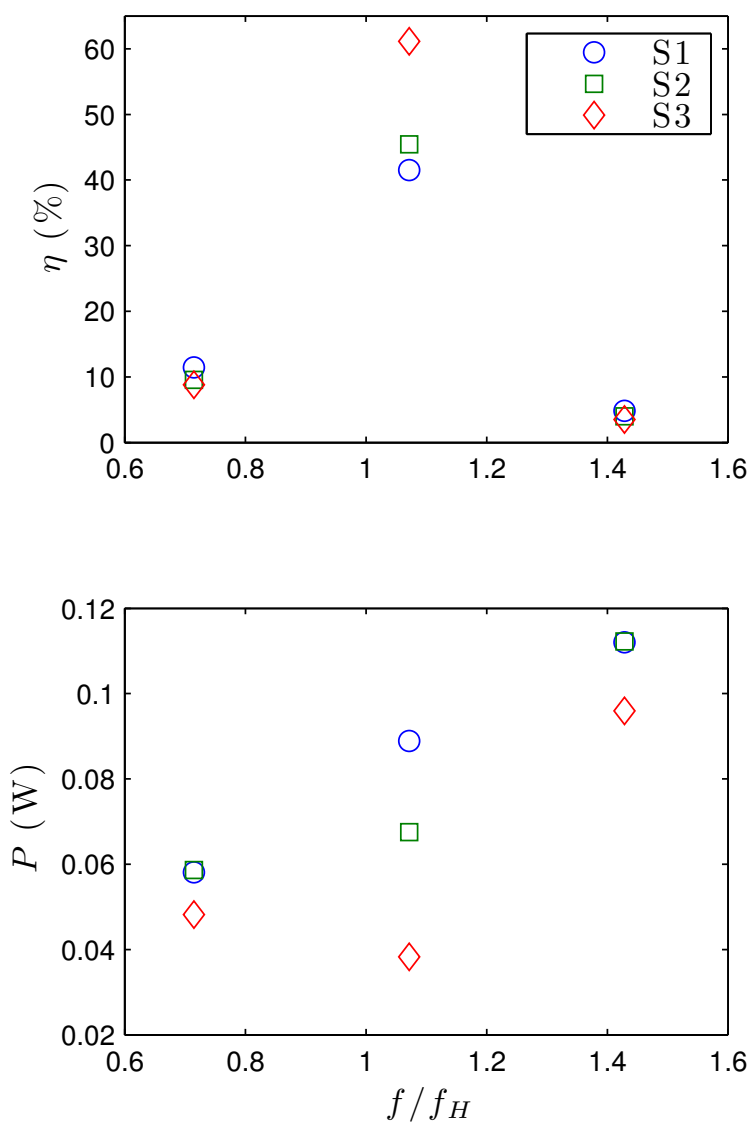

Figure 16: Fluidic efficiency and electrical power consumption as a function of $f / f_{H}$.

The preceding discussion highlights the difficulty in identifying which factor causes the improved efficiency for S3: lower electrical power consumption due to a lower Helmholtz frequency, or higher cavity pressure at the resonant peak. Comparing $\eta$ for S1 and S2 at $f / f_{H}=1.07$ demonstrates that despite the difference in cavity pressure (and therefore voltage amplitude) and Reynolds number, the efficiency varies by only $3 \%$. It can be surmised that the increased efficiency for S3 at the resonant peak is due to the combination of a lower 


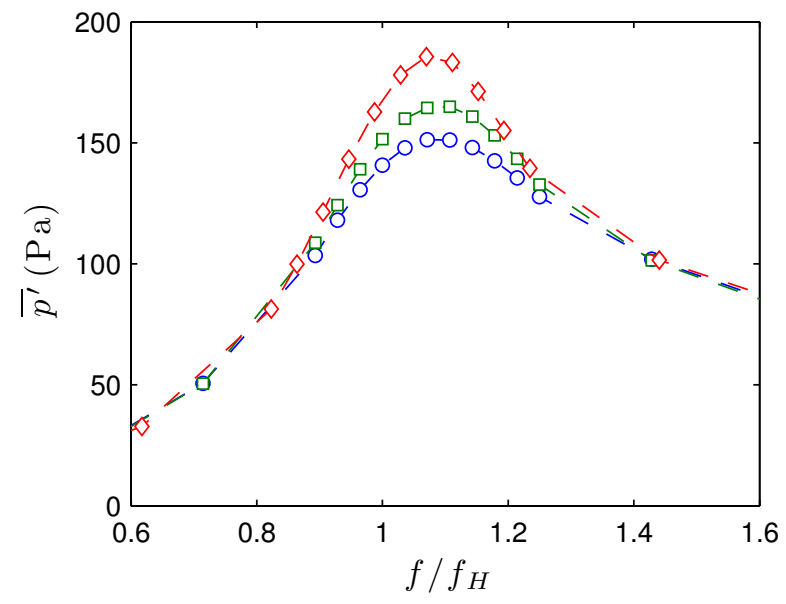

Figure 17: RMS cavity pressure for $E=250 \mathrm{~V}$ in the region surrounding the resonant peak at $f / f_{h}=1.07$. Markers indicate (o) S1, ( $\square)$ S2 and $(\diamond)$ S3.

applied voltage and lower current draw. These results demonstrate that when comparing piezoelectrically driven synthetic jets with similar performance, the most efficient design will be the one with the lowest resonant frequency. The contraction shape of S3 yields the lowest momentum flux, however it achieves significantly larger efficiency at the resonant peak. This measure of fluidic efficiency does not account for other factors that may be important in the overall design, such as the physical dimensions of the synthetic jet which are often of great concern when installing the actuator in an aerodynamic body. Table 2 qualitatively compares several important parameters of the synthetic jets: $R e_{\bar{U}}, J, \eta$ at the resonant peak near $f_{H}$ and the height of the cavity. Geometries S2 and S3 are compared to the baseline cylindrical cavity, S1, where "-" indicates negligible change (less than 10\%). The results in this table demonstrate that if the goal of a particular synthetic jet design is to maximize performance, the ideal choice would be the baseline cylindrical cavity shape since it achieves the largest momentum flux with the smallest cavity height. If the goal is to maximize efficiency, the contraction cavity provides substantially higher efficiency provided it is operated at the resonant peak corresponding to Helmholtz resonance of the cavity. 
Table 2: Comparison of geometries S2 and S3 relative to S1 based on Reynolds number, momentum flux, efficiency at resonance and cavity height.

\begin{tabular}{ccccc}
\hline \hline Geometry & $R e_{\bar{U}}$ & $J$ & $\eta_{f \approx f_{H}}$ & Cavity Height \\
\hline S2 & - & $\Downarrow$ & - & $\Uparrow$ \\
\hline S3 & $\Downarrow$ & $\Downarrow$ & $\Uparrow$ & $\Uparrow$ \\
\hline
\end{tabular}

\section{Conclusion}

The influence of cavity shape on synthetic jet performance was investigated experimentally by comparing three cavity shapes: cylindrical, conical and contraction. Characterization of the synthetic jets revealed two resonant peaks in the response of both centreline velocity and cavity pressure to excitation voltage. Due to differences in the resonant peak associated with Helmholtz resonance of the cavity, a velocity-pressure model was adopted in order to ensure equivalent operating conditions for the difference cavity shapes. Three test cases were considered at frequencies near the Helmholtz resonance. For each test case, the synthetic jets were operated with equivalent $f / f_{H}$ and cavity pressure.

Radial profiles of $U_{j}$ at the exit plane demonstrated noticeable differences in the velocity profile throughout the excitation cycle for the three cavity shapes. More precisely, the profiles have the same shape, but different magnitude. A sequential decrease in Reynolds number from cavity S1 to S2 to S3 was observed. Velocity profiles typical of fully-developed oscillatory flow were observed during the expulsion stroke for Stokes numbers from 17 to 25 . The expected zero-net-mass-flux behaviour was confirmed to be within $11 \%$ at a measurement plane of $x / d=0.075$, providing further confidence in the measured velocity profiles.

The performance of the jet for flow control applications was quantified by calculating the mean momentum flux. The results demonstrated that in agreement with the velocity profiles, the largest momentum flux was achieved by the cylindrical cavity and decreased from the conical to the contraction cavity. These results provide experimental evidence that cavity shape does impact synthetic jet performance. The fluidic efficiency was also considered and due to a lower Helmholtz frequency, the contraction cavity consumed significantly less power at the resonant peak and achieved the largest efficiency at resonance. The results provide important 
information concerning synthetic jet design: if the goal is to maximize momentum flux, the basic cylindrical shape should be selected, while if the goal is to reduce power consumption and increase efficiency, a more complex geometry can be used to achieve a lower resonant frequency.

\section{Acknowledgements}

The authors gratefully acknowledge support from the Natural Sciences and Engineering Research Council of Canada.

\section{References}

[1] Amitay, M., Smith, D. R., Kibens, V., Parekh, D. E., and Glezer, A. (2001). Aerodynamic Flow Control over an Unconventional Airfoil Using Synthetic Jet Actuators. AIAA Journal, 39(3):361-370.

[2] Bell, J. H. and Mehta, R. D. (1988). Contraction Design for Small Low-Speed Wind Tunnels. NASA STI/Recon Technical Report N, 89:13753.

[3] Bruun, H. H. (1995). Hot-wire Anemometry: Principles and Signal Analysis. Oxford University Press, Oxford.

[4] Chanaud, R. (1994). Effects of Geometry on the Resonance Frequency of Helmholtz Resonators. Journal of Sound and Vibration, 178(3):337-348.

[5] Chaudhari, M., Verma, G., Puranik, B., and Agrawal, A. (2009). Frequency response of a synthetic jet cavity. Experimental Thermal and Fluid Science, 33(3):439-448.

[6] Gallas, Q. (2005). On the Modeling and Design of Zero-Net Mass Flux Actuators. PhD thesis, University of Florida.

[7] Gallas, Q., Holman, R., Nishida, T., Carroll, B., Sheplak, M., and Cattafesta, L. (2003). Lumped Element Modeling of Piezoelectric-Driven Synthetic Jet Actuators. AIAA Journal, 41(2):240-247.

[8] Glezer, A. and Amitay, M. (2002). Synthetic Jets. Annual Review of Fluid Mechanics, 34(1):503-529. 
[9] Greenblatt, D., Paschal, K. B., Chung-Sheng, Y., and Harris, J. (2006). Experimental Investigation of Separation Control Part 2: Zero Mass-Flux Oscillatory Blowing. AIAA Journal, 44(12):2831-2845.

[10] Holman, R., Utturkar, Y., Mittal, R., Smith, B. L., and Cattafesta, L. (2005). Formation Criterion for Synthetic Jets. AIAA journal, 43(10):2110-2116.

[11] Jain, M., Puranik, B., and Agrawal, A. (2011). A Numerical Investigation of Effects of Cavity and Orifice Parameters on the Characteristics of a Synthetic Jet Flow. Sensors and Actuators A: Physical, $165(2): 351-366$.

[12] Kercher, D. S., Lee, J.-B., Brand, O., Allen, M. G., and Glezer, A. (2003). Microjet Cooling Devices for Thermal Management of Electronics. Components and Packaging Technologies, IEEE Transactions on, $26(2): 359-366$.

[13] Kotapati, R. B., Mittal, R., and Cattafesta Iii, L. N. (2007). Numerical Study of a Transitional Synthetic Jet in Quiescent External Flow. Journal of Fluid Mechanics, 581:287-321.

[14] Lee, C.and Hong, G., Ha, Q. P., and Mallinson, S. G. (2003). A Piezoelectrically Actuated Micro Synthetic Jet for Active Flow Control. Sensors and Actuators A: Physical, 108(1):168-174.

[15] Nani, D. J. and Smith, B. L. (2012). Effect of Orifice Inner Lip Radius on Synthetic Jet Efficiency. Physics of Fluids, 24(11):115110.

[16] Panchapakesan, N. and Lumley, J. (1993). Turbulence Measurements in Axisymmetric Jets of Air and Helium. Part 1. Air Jet. Journal of Fluid Mechanics, 246:197-223.

[17] Panton, R. L. and Miller, J. M. (1975). Resonant Frequencies of Cylindrical Helmholtz Resonators. The Journal of the Acoustical Society of America, 57(6):1533-1535.

[18] Pavlova, A. and Amitay, M. (2006). Electronic Cooling using Synthetic Jet Impingement. Journal of heat transfer, 128(9):897-907.

[19] Persoons, T. (2012). General Reduced-Order Model to Design and Operate Synthetic Jet Actuators. AIAA journal, 50(4):916-927. 
[20] Persoons, T. and O'Donovan, T. S. (2007). A Pressure-Based Estimate of Synthetic Jet Velocity. Physics of Fluids, 19(12):128104.

[21] Shuster, J. M. and Smith, D. R. (2007). Experimental Study of the Formation and Scaling of a Round Synthetic Jet. Physics of Fluids, 19(4):045109.

[22] Smith, B. and Glezer, A. (2002). Jet Vectoring using Synthetic Jets. Journal of Fluid Mechanics, $458: 1-34$.

[23] Smith, B. L. and Glezer, A. (1998). The Formation and Evolution of Synthetic Jets. Physics of Fluids, 10:2281.

[24] Smith, B. L. and Swift, G. W. (2001). Synthetic Jets at Large Reynolds Number and Comparison to Continuous Jets. AIAA paper, 3030:2001.

[25] Tsuji, Y., Fransson, J. H., Alfredsson, P. H., and Johansson, A. V. (2007). Pressure Statistics and their Scaling in High-Reynolds-Number Turbulent Boundary Layers. Journal of Fluid Mechanics, 585:1-40.

[26] Utturkar, Y., Mittal, R., Rampunggoon, P., and Cattafesta, L. (2002). Sensitivity of Synthetic Jets to the Design of the Jet Cavity. AIAA paper, 124:2002.

[27] White, F. M. (1979). Viscous Fluid Flow. McGraw-Hill, New York.

[28] Yavuzkurt, S. (1984). A Guide to Uncertainty Analysis of Hot-Wire Data. ASME Transactions Journal of Fluids Engineering, 106:181-186. 